\section{Tiempos de atención de urgencias neurológicas en un hospital regional de alta complejidad}

\author{
ÁlVARO SOTO V. ${ }^{1,2,3}$, GLADYS MORALES I., ${ }^{3,4, a, b}$, CLAUDIO VEGA C..$^{5}$, \\ GONZALO ECHEVERRÍA V.c, MARÍA BELÉN COLINAS G. ${ }^{c}$, \\ PEDRO CANALES O. ${ }^{c}$, DANIELA CONTRERAS B. ${ }^{c}$
}

\section{Waiting times for neurological emergencies in an emergency room}

Background: Neurological emergencies constitute 10-15\% of medical emergencies. Doctor Hernán Henríquez Aravena Hospital has in house neurologists present permanently at the Emergency Room since July 2013. Aim: To estimate the waiting times for neurological consultations; to compare the waiting times between neurovascular (UV) and non-vascular (UNV) emergencies; and to compare the waiting times of two prioritization (triage) models. Material and Methods: A convenience sample of the consultations made during shift \# 1 at the emergency room between January and December 2016, was analyzed. Results: There were 859 consultations in the period, 570 for UNV and 289 for UV. Mean age of consultants was 57 years and $52 \%$ were women. The median time for having an evaluation by a neurologist was $106 \mathrm{~min}$ (132 and $81 \mathrm{~min}$ for UNV and UV respectively). Twenty seven percent of patients were evaluated in less than one hour (23 and $36 \%$ of UNV and UV, respectively). The change of the prioritization model decreased the waiting time by 81 and 32 min for UNV and UV, respectively. Conclusions: There were significant differences in waiting times between neurovascular and non-vascular emergencies. Most patients were not evaluated in less than 60 minutes. The change in the initial stratification model was associated with a significant reduction in the waiting times for neurological emergencies.

(Rev Med Chile 2018; 146: 885-889)

Key words: Emergency Service, Hospital; Neurology; Neurologists; Stroke; Triage.

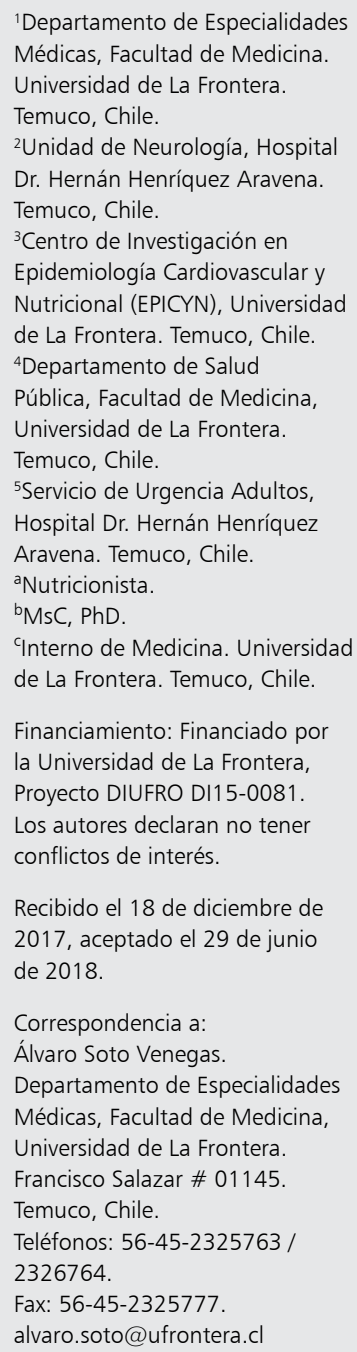

Correspondencia a:

Álvaro Soto Venegas.

Departamento de Especialidades Médicas, Facultad de Medicina,

Universidad de La Frontera.

Francisco Salazar \# 01145.

Temuco, Chile.

Teléfonos: 56-45-2325763/

2326764.

Fax: 56-45-2325777.

alvaro.soto@ufrontera.cl

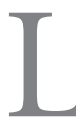

as urgencias neurológicas constituyen $10-15 \%$ de las urgencias médicas ${ }^{1}$. Se aso$\checkmark$ cian a un mayor riesgo vital y de secuelas, comparadas con otras emergencias médicas ${ }^{2}$. En muchas patologías neurológicas, como ataque cerebrovascular (ACV), meningitis bacteriana y status epilepticus, el diagnóstico rápido y certero es de suma importancia, debido a que el pronóstico depende de la velocidad con la que se inicie el tratamiento específico ${ }^{3-5}$.

Se ha sugerido que los pacientes con una urgencia neurológica vital (status epilepticus, hemorragia subaracnoidea, etc.) sean evaluados por neurólogo(a) en un tiempo inferior a 15 min desde su llegada, y los sujetos con otras urgencias neurológicas sean valorados en un tiempo inferior a $45 \mathrm{~min}^{6}$. Dentro de los factores asociados a una evaluación más rápida, en sujetos con ACV agudo, se encuentra la llegada de los pacientes en ambulancia y la admisión de estos a unidades de paciente crítico ${ }^{7}$.

En una revisión sistemática sobre tiempos de atención en ACV agudo, el tiempo llegada-evaluación por neurólogo alcanzó una mediana de 0,2 a $3,1 \mathrm{~h}^{8}$. 
En el Hospital Dr. Hernán Henríquez Aravena (HHHA) de Temuco se utiliza un sistema de categorización de riesgo (triage) de cinco niveles $(\mathrm{Cl}$ a C5), denominado selector de demanda ${ }^{9,10,11}$. Hasta abril de 2016 se realizaba triage por enfermería, seguido por una primera atención por un médico general y evaluación por especialista según criterios de derivación (Modelo 1). Posteriormente, se eliminó el apoyo del médico general. Por lo tanto, tras la categorización se realiza una atención preferencial por la especialidad que corresponda (Modelo 2).

El HHHA cuenta con neurólogos presenciales permanente en el Servicio de Urgencia de Adultos (SUA) desde julio de 2013, y posee 730 camas $^{12}$. Es el único hospital de alta complejidad de la Región de la Araucanía, y atiende a una población beneficiaria de aproximadamente 800 mil habitantes.

Los objetivos de este estudio son: estimar los tiempos de atención de las consultas neurológicas, comparar los tiempos de atención entre urgencias neurovasculares (UV) y no vasculares (UNV), y comparar dos modelos de atención inicial y su efecto sobre los tiempos para la evaluación por neurólogo(a).

\section{Material y Método}

El estudio fue prospectivo y observacional. Se seleccionó una muestra no probabilística, por conveniencia, de las consultas realizadas en el Turno $\mathrm{N}^{\circ}$ 1, del SUA-HHHA, entre enero y diciembre de 2016.

Se consideraron los antecedentes sociodemográficos (edad, sexo y etnia). Se registró categorización, diagnóstico principal y destino del paciente. Se estimaron las medianas de los tiempos de atención, medidos en minutos: tiempo llegada-priorización y tiempo llegada-evaluación por neurólogo(a).

El análisis estadístico se realizó con el software STATA 14.0. Las variables continuas se describieron con medidas de tendencia central y dispersión, promedio \pm desviación estándar (DE) o medianas con rangos intercuartílicos (RIQ). Se utilizó test $\chi^{2}$ para comparar los porcentajes del tiempo para evaluación según el modelo de atención. Se aplicó el test de la U de Mann-Whitney para asociar las medianas del tiempo para evaluación según modelo de atención.

\section{Resultados}

En la Tabla 1 se muestran las 859 consultas del período, de las cuales 289 fueron UV y 570 UNV. Se encontró una asociación estadísticamente significativa entre UV y las variables: edad, sexo masculino, etnia mapuche y ruralidad. La mediana del

Tabla 1. Descripción de la muestra, según tipo de urgencia neurológica

\begin{tabular}{|lccc|}
\hline Parámetros & Vasculares & $\begin{array}{c}\text { Urgencias neurológicas } \\
\text { No vasculares } \\
\text { (n= 570) }\end{array}$ & $\begin{array}{c}\text { Total } \\
\text { (n = 859) }\end{array}$ \\
\hline Edad, promedio \pm DE & $70,9 \pm 12,3$ & $50,6 \pm 21,3$ & $57,5 \pm 21,1$ \\
\hline Hombres, \% & 56,1 & 44,6 & 48,3 \\
\hline Etnia mapuche \% & 16,6 & 11,4 & 13,2 \\
\hline Rural, \% & 24,9 & 13 & 17 \\
\hline Tiempo priorización, minutos, Md (p75-p25) & $13,5(25-8)$ & $14(27-8)$ & $14(17-8)$ \\
\hline Tiempo evaluación, minutos, Md (p75-p25) & $81(157-35)$ & $132(250-61)$ & $106(219-55)$ \\
\hline Tiempo evaluación, minutos, promedio \pm DE & $123,6 \pm 138,7$ & $184,8 \pm 181,8$ & $164,2 \pm 170,9$ \\
\hline Evaluación < 60 min, \% & 36 & 22,6 & 27,1 \\
Categorización, \% & & & 4,7 \\
C1 & 7,6 & 3,2 & 23 \\
C2 & 32,5 & 18,1 & 71 \\
C3 & 60 & 76,6 & 1,4 \\
C4-C5 & 0 & 2,2 & \\
\hline
\end{tabular}

Md: mediana; p75: percentil 75; p25: percentil 25. 
Tabla 2. Comparación de los porcentajes de evaluación menor a 60 min en los diagnósticos de urgencias neurológicas según los modelos de atención

\begin{tabular}{|c|c|c|c|}
\hline & \multicolumn{2}{|c|}{ Evaluación < 60 min, \% } & \multirow[t]{2}{*}{ Valor $p$} \\
\hline & M1 $(n=249)$ & $M 2(n=610)$ & \\
\hline \multicolumn{4}{|l|}{ Urgencias vasculares $(n=289)$} \\
\hline Hemorragia subaracnoidea $(n=9)$ & 66,7 & 50 & n.s \\
\hline Ataque isquémico transitorio $(\mathrm{n}=26)$ & 11,1 & 29,4 & n.s \\
\hline Hemorragia intracerebral $(n=39)$ & 50 & 52,2 & n.s \\
\hline Infarto cerebral $(n=215)$ & 25,6 & 38,7 & $<0,01$ \\
\hline \multicolumn{4}{|l|}{ Urgencias no vasculares $(n=570)$} \\
\hline Vértigo $(n=28)$ & 0 & 18,2 & n.s \\
\hline No neurológico $(n=30)$ & 0 & 22,7 & n.s \\
\hline Delirium $(n=59)$ & 7,7 & 21,7 & n.s \\
\hline Trastorno neurológico funcional $(n=77)$ & 15 & 28,1 & n.s \\
\hline Epilepsia ( $n=95)$ & 24 & 45,7 & $<0,05$ \\
\hline Cefalea $(n=120)$ & 14,3 & 18,5 & n.s \\
\hline $\operatorname{Otros}(n=161)$ & 9,7 & 23,7 & $<0,05$ \\
\hline Total $(n=859)$ & 19,3 & 30,3 & $<0,001$ \\
\hline
\end{tabular}

Abreviaciones: M1. Modelo 1, M2. Modelo 2. Se aplicó test de $\chi^{2}$.

tiempo llegada-priorización fue de 14 min (RIQ: 17-8). El 4,7\% fue categorizado como C1; $23 \%$ como C2; 71\% como C3; $1,2 \%$ como C4 y $0,2 \%$ como C5. La mediana del tiempo llegada-evaluación fue de 106 min (RIQ: 219-55), 132 min para UNV y 81 min para UV. El $27,1 \%$ de los sujetos fue evaluado en menos de una hora: $22,6 \%$ con UNV y $36 \%$ con UV.

En la Tabla 2 se muestra la comparación de los porcentajes de evaluación menor a 60 min según diagnóstico y modelo de atención. En el Modelo 2 versus el Modelo 1 se registraron los mayores porcentajes de evaluación menor a $60 \mathrm{~min}$ en los diagnósticos de infarto cerebral (38,7\% y $25,6 \%)$, epilepsia $(45,7$ y $24,0 \%)$ y otros $(23,7 \%$ y $9,7 \%)$ con un $\mathrm{p}<0,05$.

La asociación entre las medianas del tiempo de evaluación según diagnóstico y modelo de atención se detalla en la Tabla 3. El cambio de modelo se asoció a una disminución de 31,5 min $(-70 \%)$ para UV y de $81 \mathrm{~min}(-56,7 \%)$ para UNV, en la mediana del tiempo de llegada-evaluación, con diferencias significativas. En particular, la mediana de este indicador para infarto cerebral bajó en 45 min $(-63 \%$; p <0,01). Al considerar la categorización, el cambio de modelo se asoció a una reducción de 7 min $(-41,2 \%)$ para $\mathrm{C} 1$, de 47 min $(-43,9 \%)$ para C2 y de $63,5 \min (-36,8 \%)$ para C3.

\section{Discusión}

Una gran proporción de las urgencias neurológicas son tiempo-dependientes ${ }^{3-5}$, por esto se recomienda que "cualquier paciente con un problema neurológico urgente pueda ser atendido por un neurólogo en menos de una hora del inicio de los síntomas"6. Idealmente, en urgencias neurológicas de riesgo vital, la evaluación debiera realizarse dentro de 15 min desde el ingreso ${ }^{6}$. En nuestro estudio, solo $27,1 \%$ de los pacientes fue evaluado en menos de una hora, con una diferencia significativa entre UV y UNV (22,6 versus $36 \%$ ). Además, con el cambio de modelo de atención el porcentaje de sujetos evaluados dentro de una hora desde su llegada aumentó de 19,3 a $30,3 \%$. Dentro de las posibles causas del bajo cumplimiento de la recomendación destacan: colapso del servicio de urgencia (overcrowding), no disponibilidad de box de atención, retraso para la categorización, retraso entre priorización 
Tabla 3. Asociación entre las medianas del tiempo de evaluación según diagnóstico y modelo de atención

\begin{tabular}{|c|c|c|c|c|}
\hline & \multicolumn{2}{|c|}{$\begin{array}{l}\text { Tiempo de evaluación } \\
\text { Md, (minutos) }\end{array}$} & \multirow[t]{2}{*}{$\begin{array}{l}\text { Diferencia } \\
\text { M2-M1 }\end{array}$} & \multirow[t]{2}{*}{ Valor $p$} \\
\hline & M1 $(n=249)$ & $M 2(n=610)$ & & \\
\hline \multicolumn{5}{|l|}{ Diagnósticos vasculares } \\
\hline Hemorragia subaracnoidea $(n=9)$ & 16 & 62,5 & 46,5 & n.s \\
\hline Ataque isquémico transitorio $(n=26)$ & 128 & 71 & -57 & n.s \\
\hline Hemorragia intracerebral $(n=39)$ & 58 & 55,5 & $-2,5$ & n.s \\
\hline Infarto cerebral $(n=215)$ & 121,5 & 76,5 & -45 & $<0,01$ \\
\hline Total vascular $(n=289)$ & 103,5 & 72 & $-31,5$ & $<0,001$ \\
\hline \multicolumn{5}{|l|}{ Diagnósticos no vasculares } \\
\hline Vértigo $(n=28)$ & 209,5 & 188 & $-21,5$ & n.s \\
\hline No neurológico $(n=30)$ & 198,5 & 133 & $-65,5$ & n.s \\
\hline Delirium $(n=59)$ & 254 & 97,5 & $-156,5$ & n.s \\
\hline Trastorno neurológico funcional $(n=77)$ & 193,5 & 105 & $-88,5$ & n.s \\
\hline Epilepsia $(n=95)$ & 135 & 66,5 & $-68,5$ & n.s \\
\hline Cefalea $(n=120)$ & 188,5 & 127 & $-61,5$ & n.s \\
\hline Otros $(n=161)$ & 202 & 115 & -87 & n.s \\
\hline \multicolumn{5}{|l|}{ Total } \\
\hline Total vascular $(n=289)$ & 103,5 & 72 & $-31,5$ & $<0,001$ \\
\hline Total no vascular $(\mathrm{n}=570)$ & 187 & 106 & -81 & \\
\hline \multicolumn{5}{|l|}{ Categorización } \\
\hline $\mathrm{C} 1$ & 17 & 10 & -7 & n.s \\
\hline $\mathrm{C} 2$ & 107 & 60 & -47 & $<0.05$ \\
\hline C3 & 172,5 & 109 & $-63,5$ & $<0,05$ \\
\hline
\end{tabular}

Abreviaciones: M1 = Modelo 1; M2 = Modelo 2. Se aplicó test de la U de Mann-Whitney.

y evaluación por neurólogo(a), categorización errónea, etc.

El Modelo 2 logró disminuir significativamente los tiempos para la evaluación por neurólogo(a). Por ejemplo, en ACV isquémico la mediana para la evaluación disminuyó en $45 \min (\mathrm{p}<0,01)$. A su vez, el porcentaje de sujetos con ACV isquémico evaluados dentro de una hora aumentó de 25,6 a 38,7\%.

En conclusión, existen diferencias significativas en los tiempos de atención entre las UV y UNV. La gran mayoría de los pacientes no son evaluados en menos de $60 \mathrm{~min}$. El cambio del modelo de atención se asoció a una disminución significativa de los tiempos de atención de urgencias neurológicas, principalmente para UV.

\section{Referencias}

1. Casado V. Atención al paciente neurológico en los Servicios de Urgencias. Neurología 2011; 26: 233-8.

2. Ara JR, Marrón R, Torné L, Jiménez A, Sánchez B, Povar J, et al. Características de la demanda de atención neurológica urgente en un hospital terciario. Estudio mediante el sistema español de triage. Neurología 2007; 22: 811-2.

3. Lees KR, Bluhmki E, von Kummer R, Brott TG, Toni D, Grotta JC, et al. Time to treatment with intravenous alteplase and outcome in stroke: an updated pooled analysis of ECASS, ATLANTIS, NINDS, and EPITHET trials. Lancet 2010; 375: 1695-703.

4. Saver JL, Smith EE, Fonarow GC, Reeves MJ, Zhao X, Olson D, et al on behalf of the GWTG-Stroke Steering 
Committee and Investigators. The "Golden Hour" and Acute Brain Ischemia. Stroke 2010; 41: 1431-9.

5. Hill CE, Parikh AO, Ellis C, Myers JS, Litt B. Timing Is Everything: Where Status Epilepticus Treatment Fails. Ann Neurol 2017; 82:155-65.

6. Sociedad Española de Neurología. Plan estratégico nacional para el tratamiento integral de las enfermedades neurológicas. Mayo 2002. Disponible en: http://www. sen.es/pdf/planacional.pdf [Consultado el 28 de octubre de 2017].

7. Lacy CR, Suh DC, Bueno M, Kostis JB. Delay in Presentation and Evaluation for Acute Stroke: Stroke Time Registry for Outcomes Knowledge and Epidemiology (S.T.R.O.K.E.). Stroke 2001; 32:63-9.

8. Evenson KR, Foraker RE, Morris DL, Rosamond WD. A comprenhensive review of prehospital and in-hospital delay times in acute stroke care. Int J Stroke 2009; 4:
187-99.

9. Escala Categorización o Selección de Demanda en Unidades de Emergencia del País, 2012. Modifica la escala 1-4 en decreto de 2009. Ordinario No 11, 2012 de la Subsecretaría de Redes Asistenciales, Minsal.

10. Acuña D, Lara B, Basaure C, Navea O, Kripper C, Saldías F. Estratificación de riesgo (Triage) en el Servicio de Urgencia. Revista Chilena de Medicina Intensiva 2015; 30 (2): 79-86.

11. Santelices E, Santelices JL. Descripción y análisis del sistema de red de urgencia (RDU) en Chile. Recomendaciones desde una mirada sistémica. Rev Med Clin Condes 2017; 28 (2): 186-98.

12. Soto A, Morales G, Pollak D, Jara V. Análisis de las consultas neurológicas en el Servicio de Urgencia de un hospital terciario. Rev Chil Neuro-Psiquiat 2016; 54 (2): 93-101. 